\title{
Transtornos mentais e comportamentais no sistema de informações hospitalares do SUS: perspectivas para a enfermagem
}

\author{
MENTAL AND BEHAVIORAL DISTURBANCES IN THE HOSPITAL INFORMATION SYSTEM: \\ PERSPECTIVES FOR NURSING
}

\section{TRASTORNOS MENTALES Y DE COMPOTAMIENTO EN EL SISTEMA DE INFORMACIONES HOSPITALARIAS DEL SUS: PERSPECTIVAS PARA LA ENFERMERÌA}

\section{Edilaine Cristina da Silva ${ }^{1}$, Moacyr Lobo da Costa Junior ${ }^{2}$}

\author{
1 Enfermeira Espe- \\ cialista em Labora- \\ tório de Pesquisa da \\ Escola de Enferma- \\ gem de Ribeirão \\ Preto da Universidade \\ de São Paulo \\ (EERP-USP). Aluna \\ do Curso de Pós- \\ Graduação, nível \\ Mestrado, da EERP. \\ USP. \\ nane@eerp.usp.br. \\ 2 Professor Doutor do \\ Departamento de \\ Enfermagem \\ Psiquiátrica e \\ Ciencias Humanas \\ da EERP-USP. \\ mlobojr@eerp.usp.br
}

\section{RESUMO}

Este estudo objetivou explorar algumas das possibilidades de informações sobre os transtornos mentais e comportamentais usando a Internet, especificamente dentro do site do Ministério da Saúde, DATASUS. Apresentou elementos que ilustram recursos dos Sistemas de Informação em Saúde, os quais podem ser utilizados pelos enfermeiros como um meio seguro e simplificado para obtenção de dados. O estudo focalizou internações psiquiátricas pelo SIHSUS no município de Ribeirão Preto, SP. Ante a necessidade do enfermeiro de utilizar os recursos que possam contribuir para a organização dos serviços e para a análise da situação dos transtornos mentais e comportamentais, os dados do SIH-SUS apresentam-se como instrumento possível para desencadear o processo de análise, cujos resultados podem possibilitar mudanças importantes para o gerenciamento dos serviços, na busca de qualidade na assistência em saúde mental e psiquiatria.

\section{DESCRITORES}

Enfermagem psiquiátrica.

Saúde mental.

Sistema de informação hospitalar.

\section{ABSTRACT}

The purpose of this study was to explore some of the possibilities of getting information about mental and behavior disorders using the Internet, specifically at the Ministry of Health's DATASUS website. The site presented elements that illustrate the Health Information Systems' resources, which may be used by nurses as a safe and simplified way of obtaining data. The study focused on psychiatric hospitalization through the SIH-SUS in the city of Ribeirão Preto, State of São Paulo. Given the nurses' needs to use technological resources that can contribute for the organization of the service and the analysis of the situation of the mental and behavior disorders, SIH-SUS data are a possible instrument to start the analysis whose results may make possible important and effective changes for the management of the services, in search for quality in mental and psychiatric health assistance.

\section{KEY WORDS}

Psychiatric nursing.

Mental health.

Hospital information system.

\section{RESUMEN}

En este estudio se tuvo como objetivo explorar algunas posibilidades de informaciones sobre los trastornos mentales y de comportamiento usando Internet, específicamente dentro de la página Web del Ministerio de Salud, DATASUS. Se presentó elementos que ilustran recursos de los Sistemas de Información en Salud, que pueden ser utilizados por los enfermeros como un medio seguro y simplificado para la obtención de datos. El estudio enfocó hospitalizaciones psiquiátricas realizadas por el SIH-SUS en el municipio de Ribeirão Preto, estado de São Paulo. Ante la necesidad que tiene el enfermero de utilizar los recursos que puedan contribuir a la organización de los servicios y análisis de la situación de los trastornos mentales y de comportamiento, los datos del SIH-SUS se presentan como instrumento posible para desencadenar el proceso de análisis, cuyos resultados pueden posibilitar cambios importantes para la gerencia de los servicios, en búsqueda de calidad en la asistencia en salud mental y psiquiátrica.

\section{DESCRIPTORES}

Enfermería psiquiátrica.

Salud mental.

Sistema de información hospitalaria. 


\section{INTRODUÇÃO}

A informação em saúde possui importante papel nas políticas de saúde, permitindo as ações de planejamento, prevenção, promoção e recuperação, a medida que forma um sistema para aquisição, organização e análise de dados necessários à definição de problemas e riscos para a saúde e à avaliação da eficácia, da eficiência e da influência que os serviços prestados exercem sobre a saúde da população, e ainda, contribui para a produção de conhecimento acerca da saúde e assuntos relacionados ${ }^{(1)}$.

No Brasil, os Sistemas de Informação em Saúde (SIS) são responsáveis por agregar informações de abrangência nacional. Tais sistemas, possibilitam inúmeras avaliações, sejam através dos sistemas de informações assistenciais ou através dos sistemas de informações epidemiológicas ${ }^{(2)}$.

Os SIS dão subsídio para uma série de pesquisas desenvolvidas no país. Estudos estes que demonstram a amplitude dos dados disponibilizados por estes sistemas nas diversas áreas do setor saúde ${ }^{(3-10)}$.

Como parte do desenvolvimento da tecnologia de informática, os SIS, difundem-se de forma a contribuir para o avanço da atenção em saúde. Para a enfermagem, o uso dos recursos dessa tecnologia, apresenta-se como instrumento de ajuda, diante da complexidade, cada vez maior, no cuidado ao ser humano por este profissional ${ }^{(11)}$.

As internações hospitalares representam uma dentre as várias fontes de dados em morbidade, que permitem o conhecimento de importante porção da morbidade global, fornecendo dados dos indivíduos hospitalizados ${ }^{(12)}$.

Além disso, há de se admitir o fato de que as internações hospitalares são grandes consumidoras de recursos humanos, materiais e tecnológicos, do setor saúde. Dentre as internações, a despesa em psiquiatria só perde para as doenças respiratórias, representando a segunda fonte de despesa com internações hospitalares no país ${ }^{(13)}$.

Ao observar a variedade das áreas da saúde focalizadas nos estudos com base nos Sistemas de Informação em Saúde, nota-se que na enfermagem em saúde mental e psiquiatria, pouco se discute a esse respeito, indicando a necessidade de estudos que contribuam para a construção desse conhecimento. Acredita-se que um dos motivos possa ser o pouco conhecimento sobre a existência e as possibilidades que esse sistema de informação permite ao usuário.

\section{METODOLOGIA}

O objetivo deste trabalho foi apresentar à comunidade de enfermagem uma maneira simples, rápida e bastante eficiente de obtenção de dados e informações em saúde.
Considerando a estruturação dos Sistemas de Informação em Saúde, o presente estudo explorou algumas das possibilidades de informações sobre os transtornos mentais e comportamentais dentro do site DATASUS do Ministério da Saúde, via Internet. Como área de referência dos dados, utilizou-se o município de Ribeirão Preto.

Há vários sistemas dentro dos Sistemas de Informação em Saúde do Ministério da Saúde, dentre eles, de especial interesse, o Sistema de Informações Assistenciais, o Sistema de Informações Epidemiológicas e o Sistema de Informações Demográficas e Socioeconômicas. O primeiro é composto pelo Sistema de Internações Hospitalares (SIH-SUS) e pelo Sistema de Informação Ambulatorial (SIA/SUS). O segundo é sustentado pelo Sistema de Informações sobre Mortalidade (SIM), pelo Sistema de Informações sobre Nascidos Vivos (SINASC) e pelo Sistema de Agravos de Notificação (SINAN). O terceiro consta dados obtidos pelo Ministério da Saúde no Instituto de Geografia e Estatística - IBGE (dados censitádios e estimativas).

O SIH-SUS provê informações que são construídas a partir dos formulários das Autorizações de Internação Hospitalar (AIH). Tais conteúdos são processados pelo Departamento de Informática do SUS (DATASUS) e disponibilizados através de dois meios distintos: CD-ROMs - Movimento de Autorização de Internação Hospitalar - e Internet pelo site www.datasus.gov.br ${ }^{(14)}$.

Uma das principais variáveis deste sistema de informações é o diagnóstico referente à internação. Para esta variável é utilizada a Classificação Estatística Internacional de Doenças e Problemas Relacionados à Saúde da Organização Mundial da Saúde - Décima Revisão (CID-10), atualmente em vigor, onde os transtornos mentais e comportamentais estão contemplados no Capítulo V.

A CID-10, utilizada no exemplo deste trabalho, possui uma lista especial de tabulação para morbidade que contém 298 itens. É uma lista condensada, construída para servir de base para listas nacionais e para comparações entre países. Dentro desta lista de morbidade o conteúdo do Capítulo V, Transtornos mentais e comportamentais, compreende 8 agrupamentos: demência; transtornos mentais e comportamentais devidos ao uso de álcool; transtornos mentais e comportamentais devido ao uso de outras substâncias psicoativas; esquizofrenia, transtornos esquizotípicos e delirantes; transtornos de humor [afetivos]; trantornos neuróticos, transtornos relacionados com o "stress" e transtornos somatoformes; retardo mental; outros transtornos mentais e comportamentais ${ }^{(15)}$.

Diante dessas considerações, foi apresentada a exemplificação de levantamento de dados utilizando esse sistema de informações. 


\section{RESULTADOS E DISCUSSÃO}

\section{Percurso de acesso às informações.}

Utilizando-se navegadores, tais como Internet Explorer, Netscape ou Comunicator, digita-se www.datasus.gov.br, acessando-se a página principal do site.
Nesta página inicial (Figura 1) são apresentadas várias opções de consulta. Clica-se com o mouse em informações de saúde.

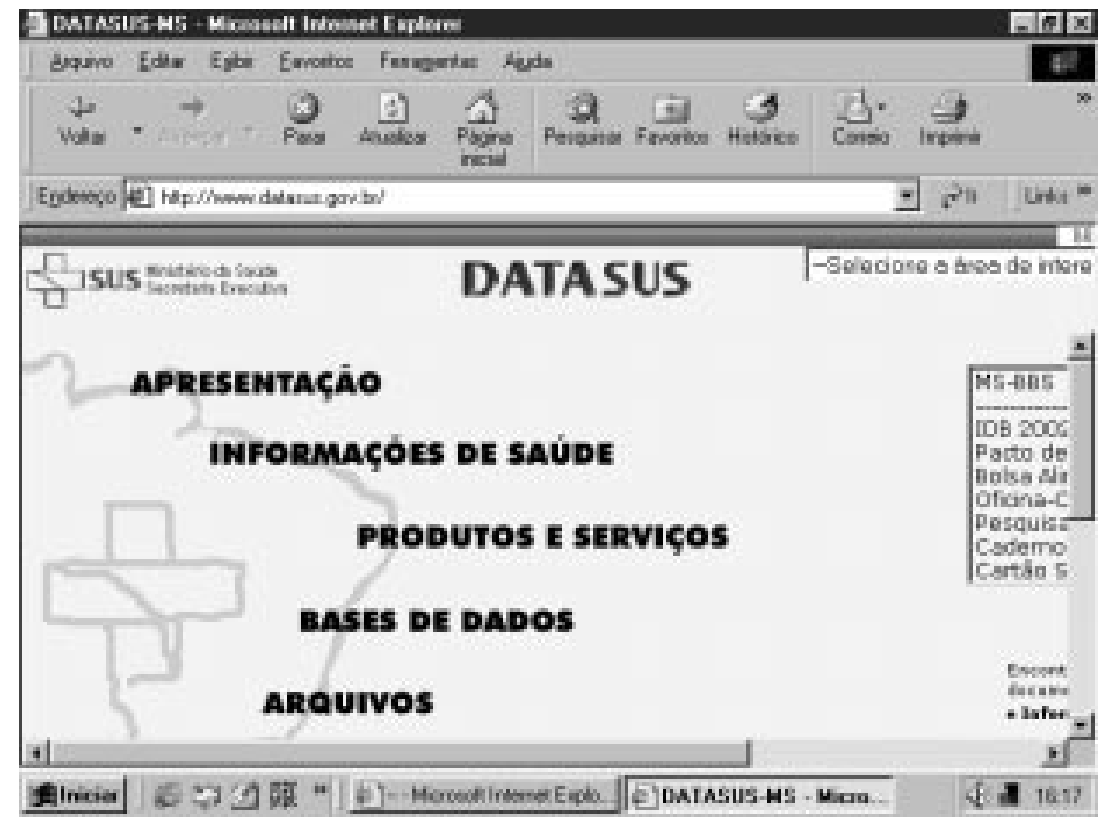

Figura 1 - Tela da página inicial de apresentação do site DATASUS

Apresentar-se-á uma nova tela com variadas opções. e Informações Epidemiológicas (Figura 2), sempre clicando Para o foco de interesse deve-se selecionar o item Morbidade sobre a opção.

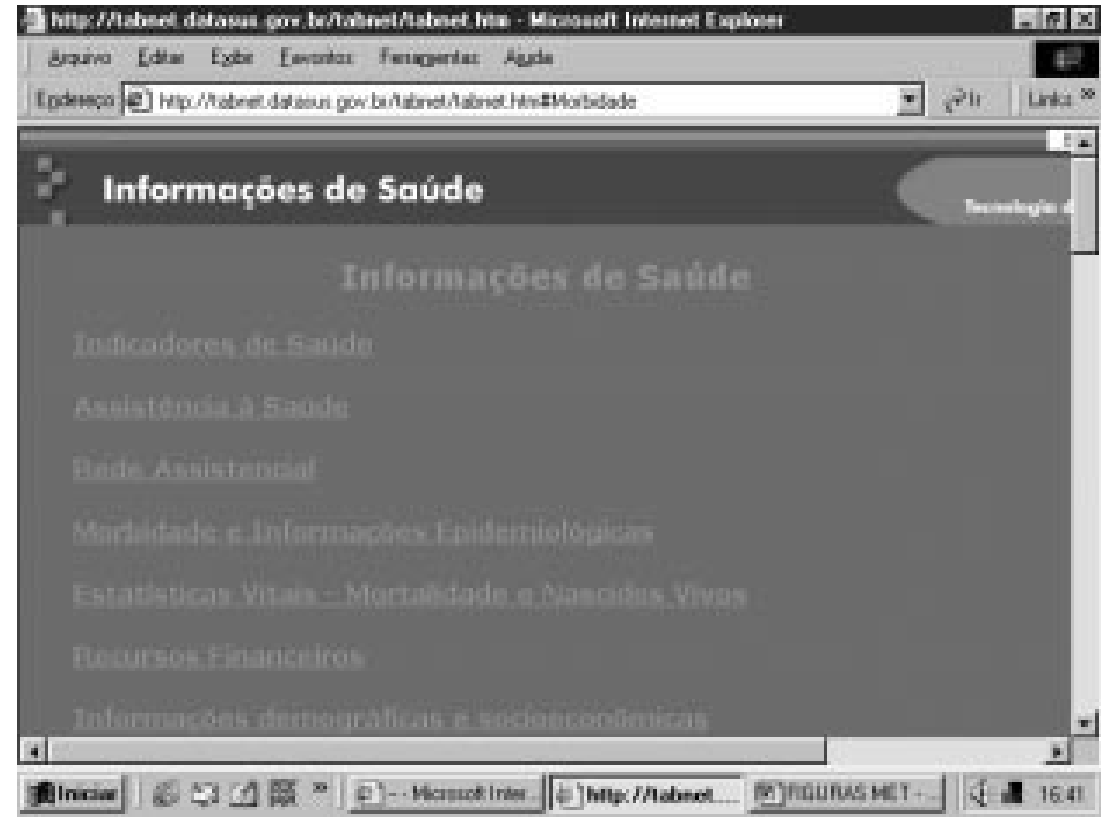

Figura 2 - Tela de I nformações de Saúde do site DATASUS 
Na sequiência, o usuário irá se deparar com duas opções para dados de morbidade (Figura 3): por local de internação (desde 1984) e por local de residência (desde 1995).

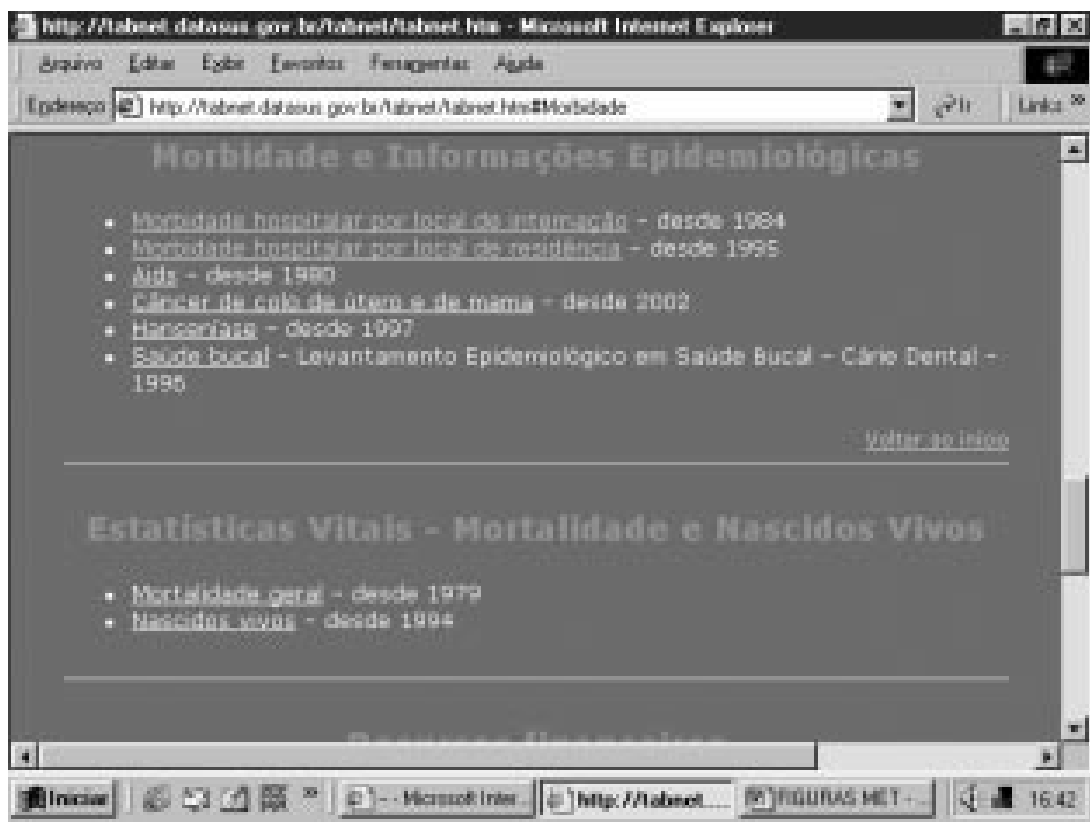

\section{Figura 3-Tela de Morbi dade el nformações E pidemi ológicas do site DATASUS}

A partir desta seleção, tem-se disponíveis variáveis como: diagnóstico através dos Capítulos 9 (até 1997) e 10 (a partir de 1998) da Classificação Internacional de Doenças e Agravos Relacionados à Saúde; tempo; idade; área de abrangência; sexo; características específicas da AIH.

Deve-se selecionar uma variável para as linhas da tabela, uma para as colunas e uma terceira para o conteúdo. Pode-se também, optar por uma só variável entre linhas e colunas. Seleciona-se o período pertinente e as demais variáveis disponíveis de acordo com o objetivo da consulta.

Após realizar todas as seleções pertinentes, clica-se sobre o ícone Mostra (Figura 4), no final da tela. As tabelas criadas podem ser impressas ou copiadas em um arquivo, como por exemplo em uma planilha de Excel. Ao serem trabalhadas em programas como o Excel, tais tabelas podem ser agrupadas resultando em quadros de consulta que resumem diversas informações.

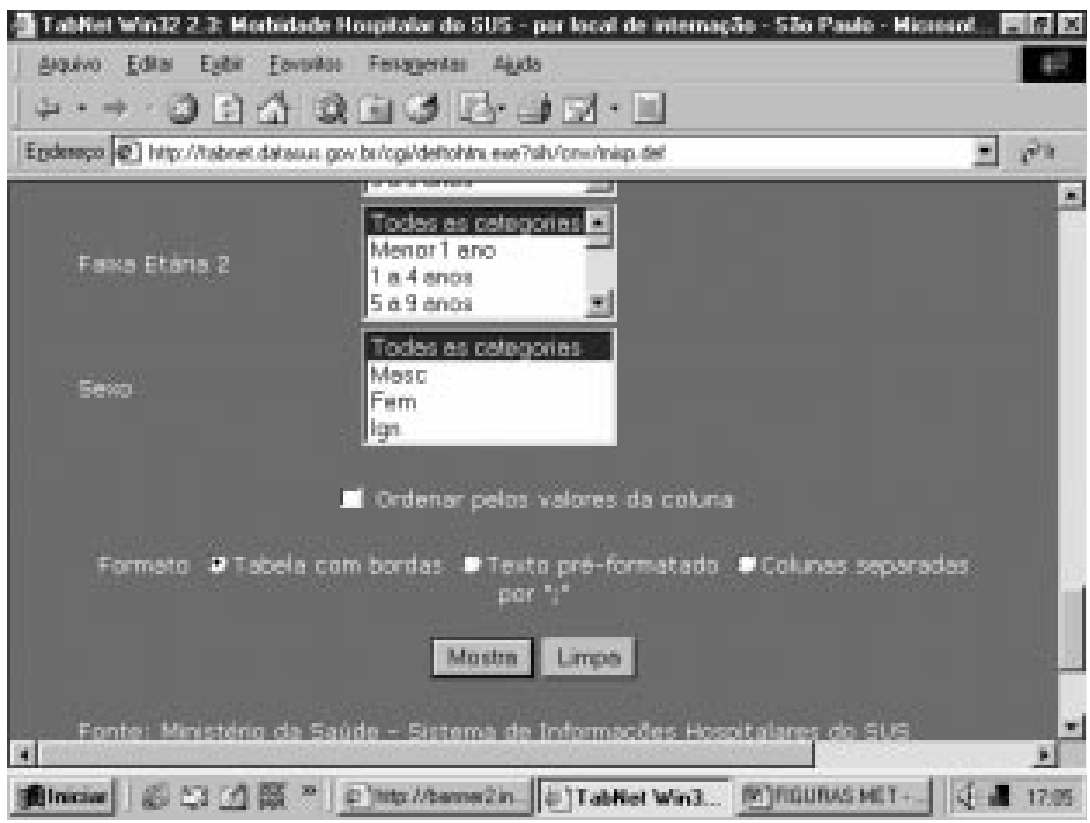

Figura 4 - Tela para sel eção de variáveis do site DATASUS 
Para busca de dados (internação, óbitos, taxa de mortalidade, AIH pagas etc) relativos aos transtornos mentais, procede-se a consulta por local de internações SUS no Município de Ribeirão Preto, de 1998 a 2002 selecionando-se em:

-Linha: Ano;Coluna: sem seleção; Conteúdo: Internações; Período: 1998 a 2002; Seleções disponíveis: Em Município $\rightarrow$ Ribeirão Preto.

Em Capítulo CID-10 $\rightarrow$ Cap. V - Transtornos mentais e comportamentais

Convém ressaltar que a CID-10 deve ser selecionada para o período a partir de 1998, sendo que para período anterior a este, selecionar-se-ia a CID-9, mantendo o Capítulo V.

Dessa forma, foi possível observar a evolução do dado no decorrer de um período, identificar crescimento, oscilação, estabilização ou regressão do número de internações psiquiátricas, tal como ocorreu neste período.

Tabela 1 - Internações Hospitalares pelo Capítulo VTranstornos Mentais e Comportamentais - da CID-10 segundo ano de ocorrência em Ribeirão Preto -1998 -2002

\begin{tabular}{cc}
\hline Ano & No de internações \\
\hline 1998 & 3285 \\
1999 & 3277 \\
2000 & 2729 \\
2001 & 2349 \\
2002 & 1881
\end{tabular}

Fonte: DATASUS, disponível em www.datasus.gov.br
Analisando os dados relativos ao Capítulo V- Transtornos mentais e comportamentais, da CID-10, verificamos que, num período de 5 anos, o número de internações no município de Ribeirão Preto apresentou uma diminuição de 42,7\%.

Outro modo de apresentar essas informações de maneira mais detalhada segundo agrupamentos recomendados pela OMS ${ }^{(13)}$, é através da lista de morbidade CID-10, como pode ser verificado na Tabela 2 . Para obtenção desta tabela devese seguir os seguintes passos:

- Linha: Lista de Morbidade CID-10; Coluna: Ano; Conteúdo: Internações; Período: 1998 a 2002; Seleções disponíveis: Em Município $\rightarrow$ Ribeirão Preto

Em Capítulo CID-10 $\rightarrow$ Cap. V - Transtornos mentais e comportamentais

A Tabela 2 permite observar, dentro dos transtornos mentais, quanto cada uma das categorias contribuiu para determinar o número de internações. Observa-se que a categoria ....Esquizofrenia, transtornos esquizotípicos e delirantes foi responsável pelo maior número de internações (5039 internações), correspondendo a 37,3\% do total. Enquanto todas as categorias mostraram diminuição no número de internações, a categoria ...Transtornos de humor [afetivos] apresentou aumento de $23,5 \%$ em relação ao início do período estudado.

Tabela 2 - Internações Hospitalares pelo Cap.V -Transtornos Mentais e Comportamentais - da CID-10 segundo Lista de Morbidade CID-10 e ano de ocorrência em Ribeirão Preto - 1998 - 2002

\begin{tabular}{lrrrrrr}
\hline Lista de Morbidade CI D-10 & 1998 & $\mathbf{1 9 9 9}$ & $\mathbf{2 0 0 0}$ & $\mathbf{2 0 0 1}$ & $\mathbf{2 0 0 2}$ & Total \\
\hline Demência & 83 & 169 & 120 & 83 & 22 & 477 \\
Transt. mentais e comportamentais dev uso ál cool & 582 & 556 & 390 & 266 & 275 & 2069 \\
Transt. mentais comport dev uso outras subst psicoat & 142 & 103 & 104 & 105 & 97 & 551 \\
Esquizofrenia trans esquizotípicos e delirant & 1318 & 1190 & 1042 & 903 & 586 & 5039 \\
Transtornos de humor (afetivos) & 486 & 532 & 501 & 536 & 572 & 2627 \\
Transt. neurot. e relacionados com stress somatof. & 86 & 89 & 60 & 62 & 46 & 343 \\
Retardo mental & 272 & 290 & 245 & 183 & 20 & 1010 \\
Outros transtornos mentais e comportamentais & 316 & 348 & 267 & 211 & 263 & 1405 \\
\hline Total & 3285 & 3277 & 2729 & 2349 & 1881 & 13521 \\
\hline
\end{tabular}

Fonte: DATASUS, disponível em www.datasus.gov.br

Ao fazer uma comparação do número de AIHs pagas pelo SUS para o Capítulo V com os demais capítulos da CID10, buscou-se dimensionar os Transtornos Mentais e Comportamentais dentro do quadro geral de diagnósticos que determinam o pagamento de uma internação hospitalar conforme apresentado na Tabela 3. Para tal, executou-se a seguinte seqüência:
- Linha: Capítulo CID-10; Coluna: sem seleção; Conteúdo: AIH pagas; Período: 2002; Seleções Disponíveis: Em Município $\rightarrow$ Ribeirão Preto 
Tabela 3 - Distribuição anual e porcentagem das AlH pagas por capítulo da CID-10 pelo Sistema de Saúde Ribeirão Preto - 2002

\begin{tabular}{lr}
\hline Capítulo CI D-10 & Al Hs \\
pagas
\end{tabular}

Fonte: DATASUS/MS.

Em Ribeirão Preto, aproximadamente 8,5\% (5117) das autorizações de internação hospitalar (AIH) pagas pelo Sistema Único de Saúde, em 2002, estão alocadas no Capítulo $\mathrm{V}$, representando o quarto lugar entre as causas que determinaram pagamento de internação hospitalar pelo SUS.

\section{CONSIDERAÇÕES FINAIS}

Informações como essas implicariam em alto custo financeiro e temporal se obtidas através da busca em relatórios das instituições ou documentos arquivados. Da forma como foram obtidas é de alcance de qualquer gestor, profissional ou pesquisador da área, para sua utilização na organização e na pesquisa da assistência psiquiátrica e de saúde mental, sem dificuldades, em pouquíssimo tempo e com custo praticamente zero.

\section{REFERÊNCIAS}

(1) Branco MAF. Sistemas de informação em saúde local. Cad Saúde Pública. 1996;12(2):267-70.

(2) Mendes ACG, Silva VA, Júnior JB, Medeiros KR, Lyra TM, Melo Filho DA, et al. Avaliação do Sistema de Informações Hospitalares - SIH/SUS como fonte complementar na vigilância e monitoramento de doenças de notificação compulsória. Inf Epidemiol SUS. 2000;9(2):67-86.

(3) Escosteguy CC, Portela MC, Medronho RA, Vasconcellos MTL. O Sistema de Informações Hospitalares e a assistência ao infarto agudo do miocárdio. Rev Saúde Pública. 2002;36(4):491-9.
Neste estudo, foram apresentados elementos que ilustram aos enfermeiros, recursos dos sistemas de informação em saúde via Internet, os quais podem ser utilizados como um meio seguro e simplificado para obtenção de dados, pois o conhecimento da situação dos transtornos mentais e comportamentais, permite aos profissionais envolvidos, formular e executar ações importantes para mudanças necessárias. Recursos assistenciais humanos, financeiros e de infraestrutura, certamente seriam melhor distribuídos seguindose esta lógica.

A criação e a apropriação pela enfermagem de instrumentos que permitam recriar ações, visando alcançar os princípios e diretrizes do SUS, coloca o profissional enfermeiro num contexto onde representa significativo papel no conjunto dos trabalhadores e parte inequívoca do sistema de produção em saúde ${ }^{(16)}$.

O presente estudo demonstrou apenas parte das possibilidades, pois este meio de obtenção de dados, apresenta variadas opções, as quais indicam cada vez mais sua potencialidade.

Contudo, a saúde mental e a psiquiatria estruturam-se dentro de um duplo contexto de transformação, onde temse por um lado as transformações do setor saúde, e por outro a reforma do sistema de assistência em saúde mental. Diante desta realidade e das vantagens observadas neste estudo, é indispensável que sistemas como o SIH-SUS seja utilizado pelos enfermeiros na busca de informações a cerca da realidade assistencial, que contribuam tanto na organização dos serviços quanto na análise da situação dos transtornos mentais e comportamentais. Assim, o reconhecimento das fontes para a busca de informações é um primeiro passo para que a enfermagem possa desencadear o processo de análise, cujos resultados podem possibilitar mudanças importantes para o gerenciamento dos serviços, na busca de qualidade da assistência em saúde mental e psiquiatria.

(4) Martins M, Travassos C, Noronha JC. Sistema de Informações Hospitalares como ajuste de risco em índices de desempenho. Rev Saúde Pública. 2001;35(2):185-92.

(5) Ferreira VMB, Portela MC, Vasconcellos MTL. Fatores associados à subnotificação de pacientes com Aids, no Rio de Janeiro, RJ, 1996. Rev Saúde Pública. 2000;34(2):70-177.

(6) Schramm JMA, Szwarcwald CL. Diferenciais nas taxas de mortalidade neonatal e natimortalidade hospitalares no Brasil: um estudo com base no Sistema de Informações Hospitalares do Sistema Único de Saúde (SIH/SUS). Cad Saúde Pública. 2000;16(4):1031-40. 
(7) Campos MR, Martina M, Noronha JC, Travassos C. Proposta de integração de dados do Sistema de Informações Hospitalares do Sistema Único de Saúde (SIH-SUS) para pesquisa. Inf Epidemiol SUS. 2000;9(1):51-8.

(8) Scatena JHG, Tanaka OY. Utilização do Sistema de Informações Hospitalares (SIH-SUS) e do Sistema de Informações Ambulatoriais (SAI-SUS) na análise da descentralização da saúde em Mato Grosso. Inf Epidemiol SUS. 2001;10(1):1930 .

(9) Lebrão ML. Determinantes da morbidade hospitalar em região do Estado de São Paulo (Brasil). Rev Saúde Pública. 1999;33(1):55-63.

(10) Gomes FA. Sistemas de Informações Hospitalares do Sistema Único de Saúde (SIH-SUS): contribuição para o estudo da mortalidade materna no Brasil [tese]. Ribeirão Preto: Escola de Enfermagem de Ribeirão Preto/USP; 2002.

(11) Évora YDM. O paradigma da informática na enfermagem [tese livre-docência]. Ribeirão Preto: Escola de Enfermagem de Ribeirão Preto/USP; 1998.

(12) Mello Jorge MHP, Gotlieb SLD. As condições de saúde no Brasil. Rio de Janeiro: Fiocruz; 2000.
(13) Savio DNA, Seidl EF, Schechtman A, Silva RC. Reestruturação da atenção em saúde mental: situação atual, diretrizes e estratégias. In: Amarante P, organizador. Psiquiatria social e reforma psiquiátrica. Rio de Janeiro: Fiocruz; 1994. p. 195-201.

(14) Brasil. Ministério da Saúde. Departamento de Informática do Sistema Único de Saúde (DATASUS) [online]. Brasília; [s.d.]. Disponível em:http:// www.datasus.gov.br. [Acesso em 17 abr. 2004].

(15) Organização Mundial da Saúde (OMS). Classificação Estatística Internacional de Doenças Relacionadas à Saúde. $10^{\mathrm{a}}$ rev. São Paulo: Centro Colaborador da OMS para Classificação de Doenças em Português/Edusp; 1996. v.2.

(16) Nichiata LYI, Takahashi RF, Fracolli LA, Gryschek ALFPL. Relato de experiência de ensino em saúde coletiva: a informática no ensino de vigilância epidemiológica. Rev Esc Enferm USP. 2003;37(3):36-43. 